OPEN ACCESS

Edited by:

Izuru Takewaki,

Kyoto University, Japan

Reviewed by:

Giacomo Navarra

Kore University of Enna, Italy

Fabrizio Paolacci,

Roma Tre University, Italy

*Correspondence:

Marco Furinghett

marco.furinghetti@unipv.it

Specialty section:

This article was submitted to

Earthquake Engineering,

a section of the journal

Frontiers in Built Environment

Received: 13 December 2019

Accepted: 06 March 2020

Published: 03 April 2020

Citation:

Furinghetti M, Lanese I and

Pavese A (2020) Experimental Assessment of the Seismic Response of a Base-Isolated Building Through

a Hybrid Simulation Technique.

Front. Built Environ. 6:33.

doi: 10.3389/fbuil.2020.00033

\section{Experimental Assessment of the Seismic Response of a Base-Isolated Building Through a Hybrid Simulation Technique}

\author{
Marco Furinghetti ${ }^{1,2 *}$, Igor Lanese ${ }^{2}$ and Alberto Pavese ${ }^{1}$ \\ ${ }^{1}$ Department of Civil Engineering and Architecture, University of Pavia, Pavia, Italy, ${ }^{2}$ European Centre for Training \\ and Research in Earthquake Engineering, EUCENTRE, Pavia, Italy
}

Base-isolated structural systems have been more and more investigated through both numerical and experimental campaigns, in order to evaluate their effective advantages, in terms of vulnerability reduction. Thanks to the lateral response of proper isolation devices, large displacement demands can be accommodated, and the overall energy of the seismic event can be dissipated, by means of hysteretic behaviors. Among the common typologies of isolators, curved surface slider devices represent a special technologic solution, with potentially high dissipative capacities, provided by innovative sliding materials. On the other hand, the overall behavior is highly non-linear, and a number of research works have been developed, aiming at the definition of the most comprehensive analytical model of such devices. The most realistic response of a base-isolated structure could be returned by a shake table test of a full-scale building. However, dimensions of the available shake tables do not allow consideration of the common load conditions, to which the isolation devices are subjected, and consequently, scaled specimens are needed, and unrealistic responses could be found. Hybrid simulations seem to solve such an issue, by accounting for an experimental substructuring, represented by a physical device tested in a testing equipment, and a numerical substructuring, consisting of a numerical model of the superstructure. Thus, a much more realistic response of the full-scale structure can be computed. In this work, the outcomes of a number of hybrid simulations have been deeply analyzed and compared to a similar numerical model. Proper non-linear constitutive laws for isolation devices have been adopted, in order to evaluate the effectiveness of design and assessment procedures, commonly adopted in real-practice applications.

Keywords: hybrid testing, base isolation, curved surface slider devices, friction coefficient, numerical and experimental substructuring

\section{INTRODUCTION}

Experimental testing has been always a fundamental aspect of the validation process, particularly in cases in which the structural or non-structural components under investigation show a complex non-linear dynamic behavior. Among the available experimental techniques, dynamic hybrid testing with substructuring has been identified by the authors as the most suitable testing technique 
because of research peculiarities. Hybrid testing, also often referred to as hybrid simulation, or hardware-in-the-loop in mechanical and automotive fields, is one of the most advanced testing techniques, nowadays well known and recognized as a powerful and cost-effective investigation option (Pegon and Pinto, 2000; Pegon and Magonette, 2002; Calabrese et al., 2015; Bursi et al., 2017). Such a technique finds its roots in the pseudo-dynamic testing technique introduced in the late 1960s by Japanese researchers (Hakuno et al., 1969), with the aim of taking advantage of both the numerical simulation and experimental testing capabilities. Within this framework, the structure or component under investigation is split into a numerical subsystem (NS) and a complementary experimentally tested physical subsystem (PS). While NS is characterized by a well-known behavior (e.g., the deck of a bridge expected to remain in the elastic range), PS is constituted by one or more elements in which uncertainties in the numerical modeling might be relevant (e.g., elements characterized by brittle and non-linear response and rate-dependent behavior such as friction). Since the complex part of the structure is physically tested in the laboratory, an explicit model of PS is not required, thus simplifying in this respect the investigation complexity. Furthermore, since the PS to be tested is only a portion of the entire structure under investigation, a bigger and often a full-scale specimen can be considered; consequently, typical compliance problems of shake table tests of big structures can be solved, by avoiding at the same time the distortions due to geometry scaling.

The test execution time rate is a key point in hybrid testing; it differentiates between pseudo-dynamic, fast, and real-time testing, with strong influence on the achievable results. A very slow test execution, with a time scale factor of the order of $\lambda=200$, can be considered for non-rate-dependent structures, such as steel or masonry partitions, in case relaxation and creep phenomena are not relevant. On the other hand, a real-time test execution should be considered when the PS is strictly rate dependent, which is typically the case of fluid viscous dampers, liquid-Tuned Mass Dampers (TMDs), etc. In between, the test execution can be somehow dynamic but slower than real time, which can be a suitable option for many applications, including base isolation devices (Lanese, 2012; Lanese et al., 2018).

While the combination of both numerical simulation and experimental testing capabilities is very appealing from the effectiveness and achievable results points of view, such combination produces a complex and heterogeneous environment. An effective management of the test definition, implementation, and execution requires good multidisciplinary knowledge and skills of numerical simulation, signal processing, actuation and mechanical systems control. In addition, further crucial aspects derive from the combination of such different environments, for example, the treatment of noisy experimental signals in a numerical model and in a solving algorithm that might result in progressive undesired oscillations and unstable overall response.

The algorithm managing the test execution and providing the step-by-step solution of the NS and computing the displacement increments to be applied to the PS must be necessarily different from typical methods used in pure numerical simulation. Among others, iterations cannot be considered to avoid spurious oscillation in the PS, stressing and damaging the physical specimen out of the real structural behavior meant to be represented.

A partitioned method (PM) suitable to treat and couple different sub-domains, thus fitting the hybrid simulation needs, was developed by Pegon and Magonette (2002). The method was developed starting from the well-known GC method (Gravouil and Combescure, 2001), while the operations sequence has been modified to allow for a parallel-tasks execution. Two separated parallel processes, obtained through the introduction of a forward prediction, are then carried out for NS and PS, while at each so-called coarse time step, the two sub-domains are coupled. This separation allows for an independent-tasks execution; a different time step can be considered for the PS and NS; this is often desirable since the PS needs a continuous test execution at the facility digital controller rate - typically about $1 \mathrm{kHz}-$ while the NS, possibly complex and non-linear, likely needs a larger time step to provide the step-by-step solution. While a numerical model of the PS is not required, an estimation of the initial stiffness of the PS matrix is needed to implement an explicit Newmark scheme, while the numerical part is treated with a semi-implicit approach. Bonelli et al. (2008) investigated the convergence and stability characteristics of the PM, while Bursi et al. (2010) proposed an enhanced variant, that is, the PM- $\alpha$, that enables the coupling of arbitrary generalized- $\alpha$ (G- $\alpha$ ) schemes endowed with numerical dissipation.

In order to ensure a correct implementation through the hybrid testing technique, all critical aspects coming from both numerical and experimental sides and from their combination need to be addressed, together with a reliable implementation of proper boundary conditions at the interface between NS and PS and, finally, a robust strategy for the verification of the results reliability assessment.

In this work, a proper framework for the hybrid simulation of a base-isolated building has been defined, in order to compute the most realistic response of a case study structure, equipped with double-concave surface slider (DCSS) devices, when subjected to earthquake excitations. Outcomes have been compared to numerical results of non-linear time history analyses of singledegree-of-freedom (SDOF) and multiple-degrees-of-freedom (MDOF) oscillators, together with reference values returned by equivalent linear elastic analyses, which represent the most used design and assessment procedures in the common practice.

\section{CASE STUDY STRUCTURE}

The case study structure consists of a six-story reinforced concrete frame building, as shown in Figure 1: the present structural system has been deeply analyzed in recent research works, by designing all members according to Italian codeconforming provisions (D.M. 17/01/2018, 2018; Cardone et al., 2017). Plan dimensions are approximately 21 and $12 \mathrm{~m}$ for $x$ and $y$ directions, respectively, and the interstory height is $3.05 \mathrm{~m}$ for all the floors, but the ground one, which has a height of $3.4 \mathrm{~m}$; consequently, the total height of the building is equal to $19 \mathrm{~m}$. 


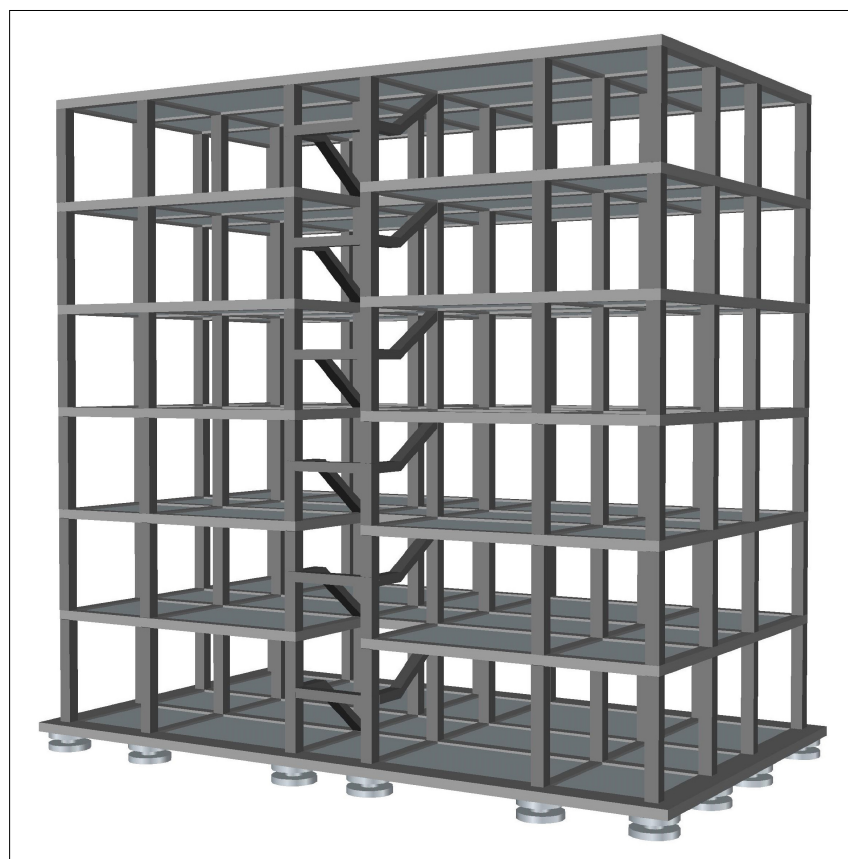

FIGURE 1 | Case study structure.

For each floor of the building, a seismic mass approximately equal to 300 tons can be considered, with a total value of 2,080 tons. According to the implemented cross sections of both beams and columns and the mass properties, the first mode of vibration of the structure is represented by a period around $1.0 \mathrm{~s}$. Modal characteristics have been deeply studied in the next sections, for the definition of the numerical substructuring of the hybrid testing framework.

\section{DEFINITION OF THE SEISMIC INPUT}

Hybrid simulations presented in this research work have been performed, by considering seven unidirectional natural seismic events (Iervolino et al., 2009; Furinghetti and Pavese, 2017). As ruled by the Italian Building Code (D.M. 17/01/2018, 2018), records have been selected according to the seismic hazard level defined for the construction site: precisely, L'Aquila has been considered as a reference location, with soil class $\mathrm{C}$ and topography category $\mathrm{T} 1$. The collapse limit state has been assumed, which corresponds to $5 \%$ probability of exceedance in the reference life of the structural system, equal to 50 years (return period 975 years). Spectrum compatibility has been checked: lower and upper bounds for the mean spectrum of the selected events have been defined according to 90 and $130 \%$, respectively, of the code design spectrum and a period range within 0.15 and $3.0 \mathrm{~s}$ has been considered. Individual ground motion records have been scaled, in order to better achieve spectrum-compatibility prescriptions; moreover, scale factors have been bounded between 0.5 and 2.0, aiming at preserving the correct frequency content for the considered peak ground acceleration (PGA) values. In Table 1, all the selected records are listed, together with the main characteristics of the considered earthquakes.

In Figure 2, results of the spectrum-compatibility check are shown, in terms of individual and mean response spectra, in comparison to the target and lower- and upper-bound graphs.

It can be noted that all the individual response spectra are significantly close to the target one: consequently, the mean spectrum fairly represents the seismic hazard level of the considered construction site.

\section{DEFINITION OF THE HYBRID TESTING FRAMEWORK}

In this section, the framework for the hybrid simulations of the case study structure is defined. Precisely, the reinforced concrete frame structure has been numerically implemented, by considering the actual stiffness and mass matrices of a full three-dimensional finite element model (FEM). The base isolation system is represented through a single physical fullscale device, which is representative of the whole set of isolators: accordingly, all isolators are assumed to be subjected to the same average vertical load, which can be computed as the total weight of the structure divided by the number of bearings, and to purely translational motion, by neglecting torsional movements of the superstructure.

\section{Experimental Substructuring}

All the hybrid simulations have been performed at the Laboratory of the EUCENTRE Foundation in Pavia (Italy) (Peloso et al., 2012). For the implementation of the experimental substructuring, the bearing tester system has been used, in order to apply and monitor the response of the full-scale physical device, which represents the whole isolation system of the case study structure. In Figure 3, the testing setup is shown.

The device is installed on a sliding bench, which applies the unidirectional translational motion, by means of two horizontal actuators. The vertical load is applied by dynamic actuators, located underneath the sliding bench, thanks to the reaction arch, which provides vertical restraint conditions. The whole testing system is governed by the laboratory hydraulic system, which consists of eight hydraulic power supply units (total flow capacity: 1,360 l pm) with 280-bar working pressure and of five accumulators banks, each one with two $45-\mathrm{L}$ piston accumulators and six 30-L gas bottles for a total piston accumulator volume of $450 \mathrm{~L}$ and a total bottle volume of $900 \mathrm{~L}$.

The device consists of a DCSS isolator (Fenz and Constantinou, 2006; De Domenico et al., 2018), with an internal non-articulated slider. Both the sliding surfaces have the same radius of curvature $(1,600 \mathrm{~mm})$, and the slider height is equal to $120 \mathrm{~mm}$ : thus, the equivalent radius of curvature results to $3,080 \mathrm{~mm}$. The implemented circular sliding pads have a diameter equal to $160 \mathrm{~mm}$, and the maximum displacement capacity is equal to $325 \mathrm{~mm}$ (Figure 4).

In order to define the vertical load to be applied at the tested device, the average reaction force has been computed, as the ratio between the total weight of the superstructure and the 
TABLE 1 | Selection of natural events.

\begin{tabular}{lcccccccc}
\hline Event \# & $\begin{array}{c}\text { Station } \\
\text { ID }\end{array}$ & $\begin{array}{c}\text { Earthquake } \\
\text { name }\end{array}$ & Date & Mw & $\begin{array}{c}\text { Fault } \\
\text { mechanism }\end{array}$ & $\begin{array}{c}\text { Epicentral } \\
\text { distance } \\
(\mathbf{k m})\end{array}$ & $\begin{array}{c}\text { Original } \\
\text { PGA (g) }\end{array}$ & $\begin{array}{c}\text { Scaled } \\
\text { PGA (g) }\end{array}$ \\
\hline 1 & ST164(x) & Kalamata & $13 / 09 / 1986$ & 5.9 & Normal & 10.0 & 0.215 & 0.429 \\
factor (\#)
\end{tabular}

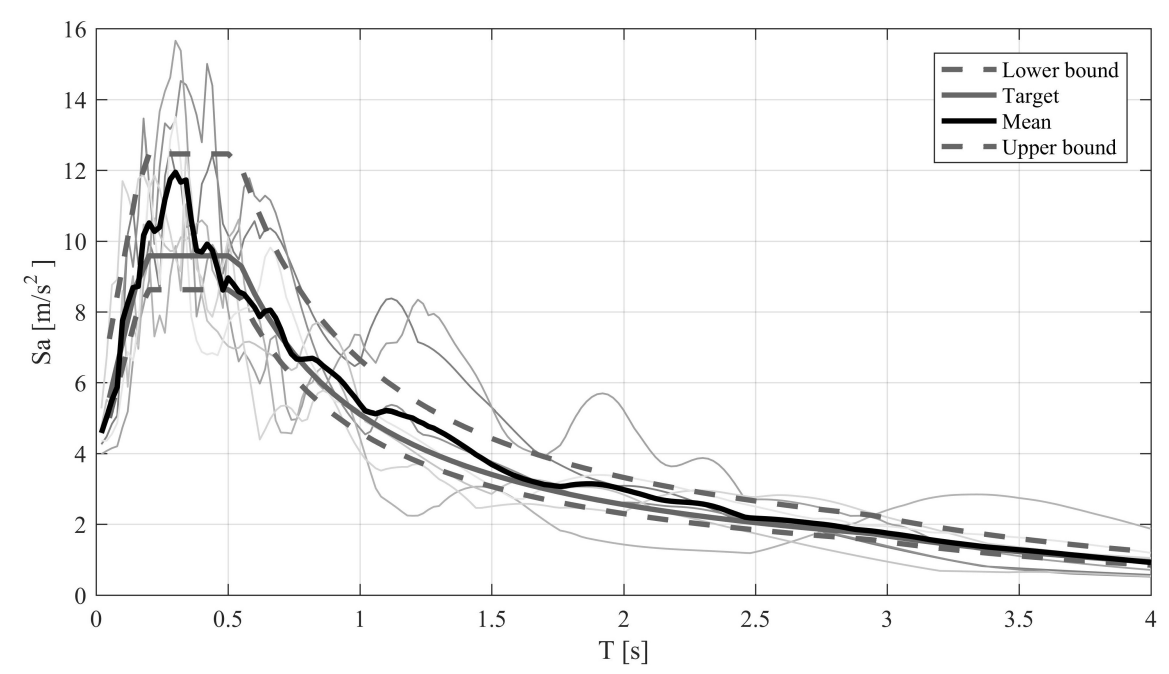

FIGURE 2 | Spectrum-compatibility graphical results.

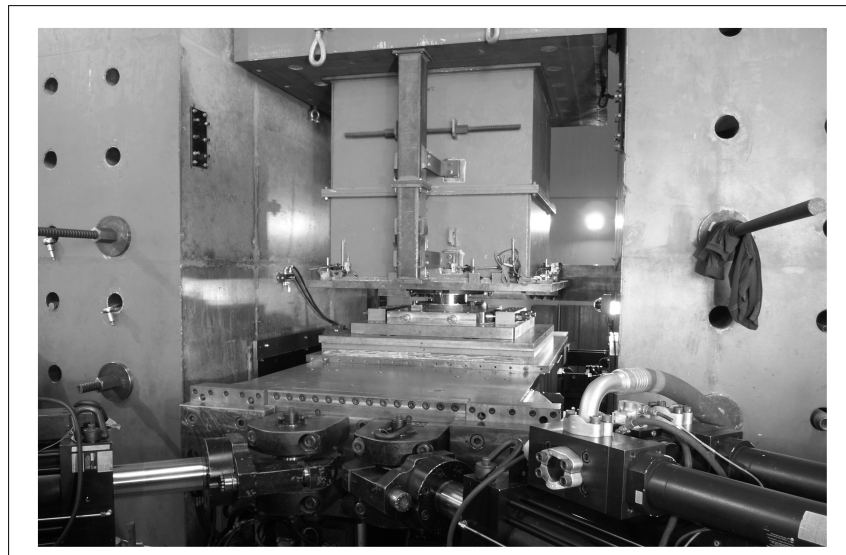

FIGURE 3 | Testing setup of the Bearing Tester System at the EUCENTRE Foundation.

number of bearings: hence with the total mass of the building at 2,080 tons and given 24 structural bearings, a vertical load of $850 \mathrm{kN}$ has been computed, corresponding to $43 \mathrm{MPa}$ of average contact pressure. The sliding pad consists of an innovative graded polytetrafluoroethylene (PTFE) material, filled with carbon fibers (Furinghetti et al., 2019a).

Firstly, frictional properties have been investigated, for a correct evaluation of the displacement demand of the isolation system and a correct definition of the time scale of the hybrid simulation (Mosqueda et al., 2004; Lomiento et al., 2013; Quaglini et al., 2014; Kumar et al., 2015; CEN, 2018). To this aim, dynamic tests have been performed on the device, by applying sinusoidal horizontal displacement time series with different frequencies (i.e., peak velocities), with a maximum displacement equal to $150 \mathrm{~mm}$ and the previously described average vertical load $(850 \mathrm{kN})$. The considered velocity levels are $2.5,10,50$, and $150 \mathrm{~mm} / \mathrm{s}$; tests at 10 and $50 \mathrm{~mm} / \mathrm{s}$ have been carried out two times, in order to assess the repeatability of the computed friction properties. In Figure 4, the resulting characterization curve is reported, together with a best-fit curve, according to the most common analytical expression of the velocity effect for PTFEbased materials (Constantinou et al., 1990; Dolce et al., 2005; Furinghetti et al., 2019a).

As expected for PTFE-based materials, the friction coefficient value achieves an asymptotic value $(8.6 \%)$ if $50 \mathrm{~mm} / \mathrm{s}$ velocity 

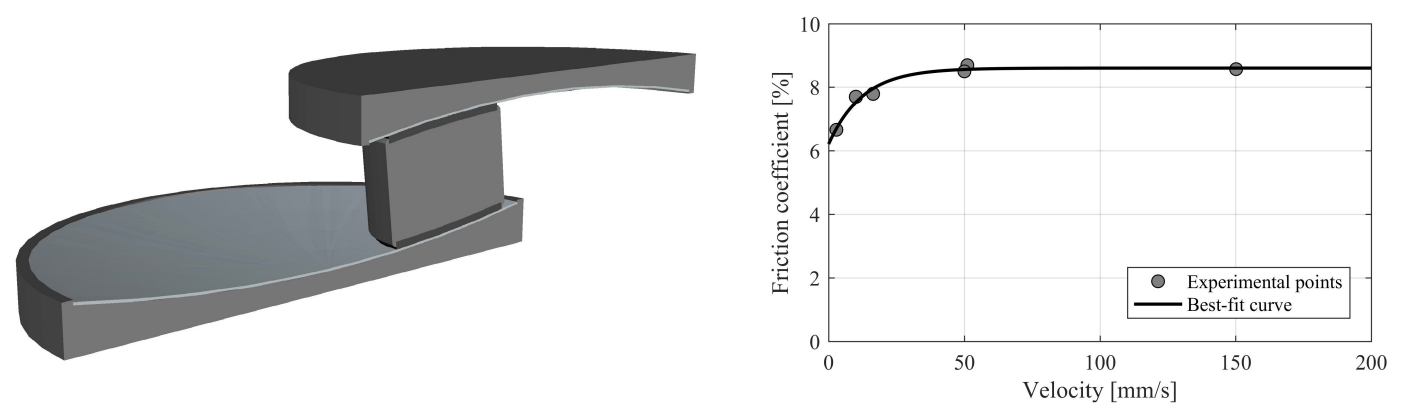

FIGURE 4 | Tested isolator and frictional characterization curve.

is exceeded. Repeated tests have provided experimental points aligned with respect to the best-fit characterization curve. Since hybrid simulations have to be carried out according to a scaled time axis and due to the just analyzed velocity effect of frictional properties, the definition of the proper time scale (TS) represents an important issue, for the correct evaluation of the response of the overall system. To this aim, an equivalent linear elastic analysis has been carried out, by considering the target acceleration displacement response spectrum (ADRS) and an equivalent linear elastic SDOF, for the computation of the seismic demands of the isolation system, in terms of maximum displacement and velocity. Such an analysis consists of an iterative procedure: initially, a numerical value of the displacement demand $D_{\max }$ is guessed, and consequently, the SDOF oscillator is characterized, by computing the following:

- the secant period of the overall isolated system:

$$
T_{s e c}=2 \pi \sqrt{\frac{D_{\max }}{g\left(\mu+\frac{D_{\max }}{R_{e q}}\right)}}
$$

- the equivalent viscous damping:

$$
\xi_{e q}=\frac{2}{\pi} \cdot \frac{\mu}{\mu+\frac{D_{\max }}{R_{e q}}}
$$

- the damping scaling factor:

$$
\eta=\sqrt{\frac{10}{5+\xi_{e q}}}
$$

- and the displacement spectral coordinate:

$$
D_{\max }=S d\left(T_{s e c}, \eta\right)
$$

Convergence is reached when the initial guessed displacement value becomes fairly comparable to the spectral coordinate, within an assumed tolerance. The spectral coordinate is computed as a function of the secant period and the damping scaling factor $\eta$ (with lower bound of 0.55 ), as ruled by the Italian Building Code (D.M. 17/01/2018, 2018).

All parameters depend on the mechanical properties of the isolation system, namely, the equivalent radius of curvature
$R_{\text {eq }}(3.08 \mathrm{~m})$ and the friction coefficient $\mu$ : at a first stage, such a parameter has been assumed equal to the asymptotic value of the characterization curve $(8.6 \%$, corresponding to a velocity higher than $50 \mathrm{~mm} / \mathrm{s}$ ), in order to evaluate the maximum velocity demand (i.e., the spectral velocity at convergence), for the correct definition of the time scale. By considering all the aforementioned hypotheses, maximum displacement and velocity demands returned by the equivalent linear elastic analysis are, respectively, equal to $150 \mathrm{~mm}$ and $447 \mathrm{~mm} / \mathrm{s}$ (secant period: $2.107 \mathrm{~s}$, equivalent viscous damping: $40 \%$ ). Thus, in order to obtain a similar frictional response in the hybrid tests, by providing simulations as close as possible to real time, a time scale factor equal to 8 has been assumed, which corresponds to $56 \mathrm{~mm} / \mathrm{s}$ peak velocity $(447 \mathrm{~mm} / \mathrm{s}$ divided by 8 ). In addition, a second set of hybrid tests have been performed, aiming at computing the response of the isolated case study structure, by considering a lower coefficient of friction: to do so, a time scale equal to 32 has been assumed, which corresponds to $14 \mathrm{~mm} / \mathrm{s}$ peak velocity and, consequently, to $7.8 \%$ of friction coefficient. In Figure 5, graphical results for equivalent linear elastic analyses are shown, for both time scale factors 32 and 8 .

In Table 2, numerical results are summarized.

Both the analyses have returned approximately the same peak velocity: hence, the definition of time scale 32 can be considered in agreement with the assumed frictional properties. In addition, other response parameters look similar between the considered cases; thus, the provided results of hybrid simulations can be interpreted as the evaluation of the response of the same structural system, equipped with two individual sliding materials (even though different time scales are considered).

\section{Numerical Substructuring}

The superstructure has been numerically modeled within the hybrid simulation algorithm, by considering an MDOF oscillator. Precisely, at each story location of the building, a single horizontal translational degree of freedom has been defined, referred to as the ground location. Aiming at considering the same behavior of the 3-D FEM of the superstructure, an ad hoc static condensation procedure has been applied and a full stiffness matrix has been computed, and consequently, the effective contribution of each column and beam is taken into account (Chopra, 1995; Furinghetti et al., 2019b). Given the full 3-D model, the location 
Time scale: 32

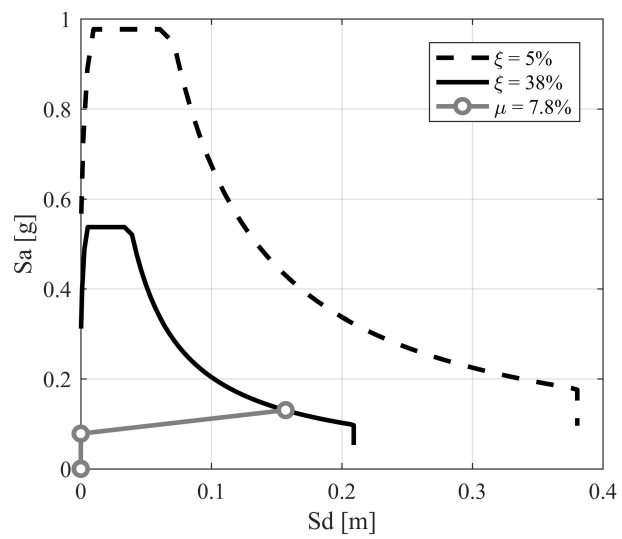

Time scale: 8

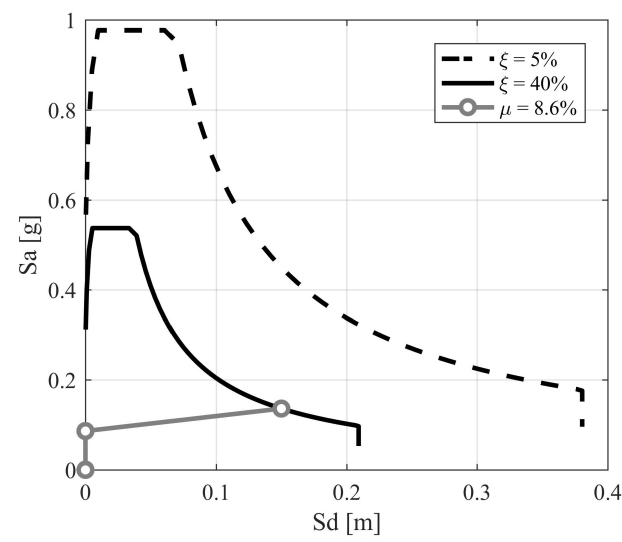

FIGURE 5 | Graphical results of equivalent linear elastic analyses.

TABLE 2 | Results of equivalent linear elastic analyses.

\begin{tabular}{|c|c|c|c|c|c|}
\hline & $D_{\max }(\mathrm{m})$ & $A_{\max }(\mathbf{g})$ & $\xi_{\text {eq }}(\#)$ & $T_{s e c}(\mathrm{~s})$ & $V_{\max }(\mathrm{m} / \mathrm{s})$ \\
\hline$\mu=7.8 \%$ & 0.157 & 0.130 & $38.1 \%$ & 2.202 & 0.448 \\
\hline$\mu=8.6 \%$ & 0.150 & 0.136 & $40.3 \%$ & 2.107 & 0.447 \\
\hline
\end{tabular}

of the center of mass has been defined for all stories, and a master-slave strategy has been adopted, by applying a rigid diaphragm constraint to all structural points of the same floor. For the computation of the ith column of the stiffness matrix, a lumped horizontal force is applied to the center of mass of the $i$ th floor, whereas all the other stories are restrained: numerical components are then obtained by dividing reaction forces by the horizontal displacement of the unrestrained floor.

Such a procedure allows consideration of the effective deformability of each story of the superstructure, from both the translational and torsional points of view, even though only translational degrees of freedom are considered: this is allowed, since the adopted concave surface slider devices generally reduce torsional effects in the response of the isolated superstructure. The resulting stiffness matrix consists of a full matrix, with no null components, far from an alternative definition of the commonly known three-diagonal matrix of a shear-type model. The mass diagonal matrix has been simply computed, by extracting for each level the assembled masses of all nodes of the considered story. In order to check the effectiveness of the proposed static condensation procedure, the modal analysis has been carried out on both the MDOF and the 3-D FEMs, by considering the fixedbase structure; results have been compared, in terms of vibration periods, modal participating mass ratios, and modal shapes. In Table 3, period and participating mass ratio values are listed for both models, whereas in Figure 6, modal shapes are analyzed.

As can be noted, modal analysis of the MDOF model have returned approximately the same values of both vibration periods and participating mass ratios of the 3-D FEM.

Also, modal shapes are approximately overlapped for all modes. The fifth and sixth modes only show small discrepancies between the compared modal shapes: nonetheless, participating mass ratios are very small, revealing a negligible contribution in the building seismic response.

Thus, the dynamic system implemented in the hybrid simulation algorithm can be expressed as follows:

$$
\begin{aligned}
& \overline{\bar{M}} \cdot\left(\begin{array}{c}
\ddot{u}_{0} \\
\ddot{u}_{1} \\
\ddot{u}_{2} \\
\vdots \\
\ddot{u}_{6}
\end{array}\right)+\overline{\bar{C}} \cdot\left(\begin{array}{c}
\dot{u}_{0} \\
\dot{u}_{1} \\
\dot{u}_{2} \\
\vdots \\
\dot{u}_{6}
\end{array}\right)+\overline{\bar{K}} \cdot\left(\begin{array}{c}
u_{0} \\
u_{1} \\
u_{2} \\
\vdots \\
u_{6}
\end{array}\right)+F_{i s}^{\exp } \cdot n_{i s} \cdot\left(\begin{array}{c}
1 \\
0 \\
0 \\
\vdots \\
0
\end{array}\right) \\
& =-\overline{\bar{M}} \cdot\left(\begin{array}{c}
1 \\
1 \\
1 \\
\vdots \\
1
\end{array}\right) \cdot \ddot{x}_{g}
\end{aligned}
$$

Where:

- $\overline{\bar{M}}, \overline{\bar{C}}$, and $\overline{\bar{K}}$ are the mass, damping, and stiffness matrices, respectively, of the whole base-isolated system;

- $u_{i}$ is the relative translational degrees of freedom located at each level of the building with respect to the ground;

- $\ddot{x}_{g}$ is the considered ground motion;

- $n_{\text {is }}$ is the number of implemented isolation bearings; and

- $F_{i s}^{e x p}$ is the experimental force of the physical substructuring, that is, the DCSS device.

The damping matrix models a multimodal damping, with 5\% for all vibration modes, and no damping for the first, second, and third modes. During the hybrid simulation, at each time 
TABLE 3 | Modal analyses comparison: vibration periods and participating mass ratios.

\begin{tabular}{|c|c|c|c|c|}
\hline Mode \# & \multicolumn{2}{|c|}{ MDOF } & \multicolumn{2}{|c|}{ FEM 3-D } \\
\hline 2 & 0.319 & $11.0 \%$ & 0.320 & $11.3 \%$ \\
\hline 3 & 0.181 & $3.7 \%$ & 0.181 & $3.7 \%$ \\
\hline 4 & 0.122 & $1.3 \%$ & 0.123 & $1.4 \%$ \\
\hline
\end{tabular}

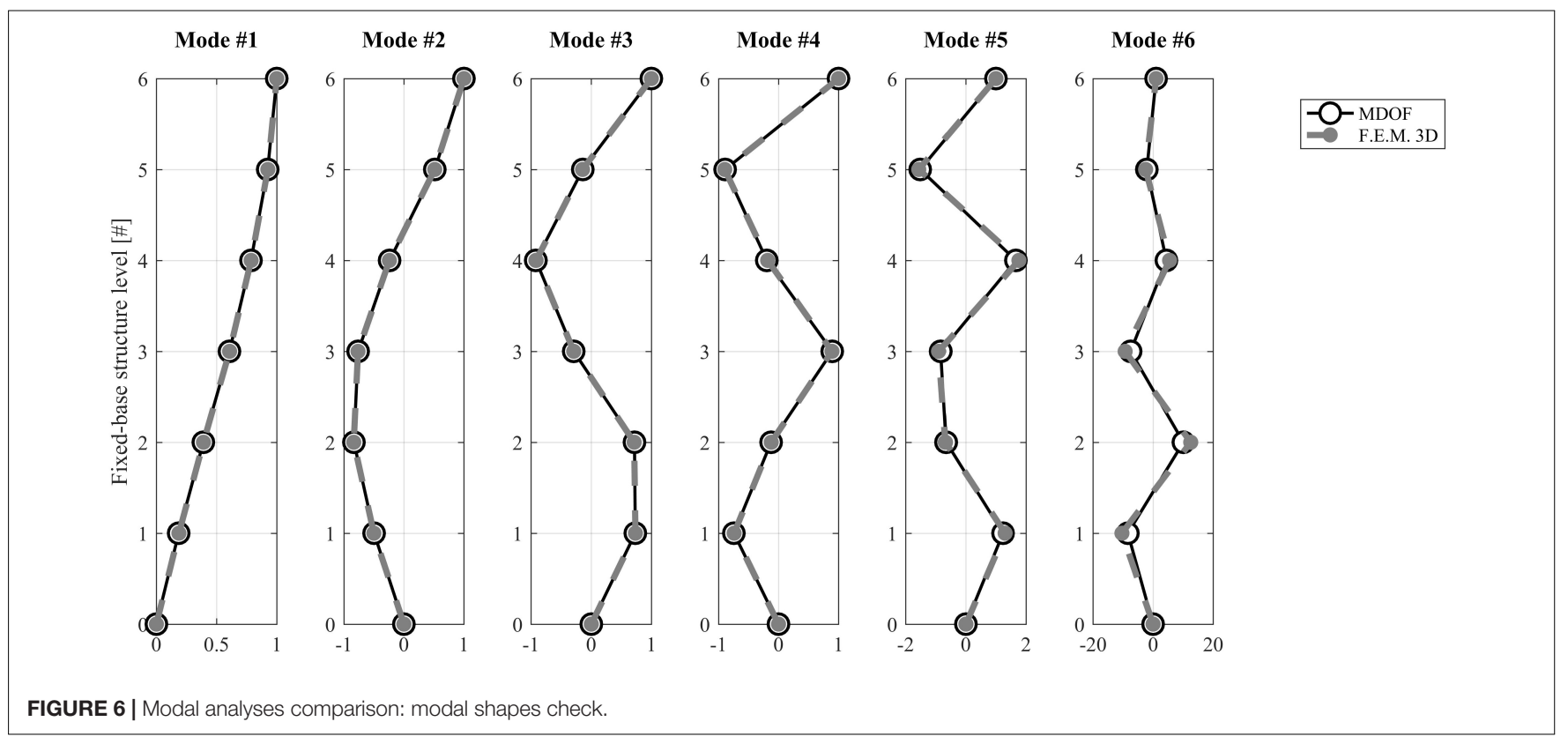

step, $u_{0}$, which is the horizontal degree of freedom at the isolation level, represents the input displacement for the bearing tester system, which applies the proper deformed shape to the physical bearing: consequently, the recorded force of the fullscale tested device $F_{i s}^{\text {exp }}$ is used in the dynamic system for the computation of all degrees of freedom at all levels. According to the assumed modeling strategy, the single full-scale tested device is representative of the force response of the whole isolation system, through the proper scale factor $n_{i s}$ and by applying the average vertical load, due to static conditions. Even though this hypothesis seems to excessively simplify the overall dynamic system, reasonable results can be obtained for the considered case study structure. Precisely, in static conditions, vertical reactions have a limited variability among all the supporting points, with standard deviation of $180 \mathrm{kN}$, related to an average value of $850 \mathrm{kN}$; the adopted sliding pad diameter $(160 \mathrm{~mm})$ implies an average contact pressure of $43 \mathrm{MPa}$, with a consequent standard deviation of $9 \mathrm{MPa}$. Thus, the frictional response is not expected to vary among the isolators, since the implemented sliding material provides a reasonable stable value of friction coefficient at the considered contact pressure range of variation [34 $\div 52 \mathrm{MPa}$ (Furinghetti et al., 2019a)]. In addition, effects of the dynamic overturning moment generally lead to negligible variation of vertical loads at isolation devices (Pavese et al., 2019), and no vertical component of the selected seismic events have been considered.

\section{RESULTS}

In what follows, results of hybrid tests are analyzed, for both the considered time scale factors. Precisely, attention has been focused on the experimental hysteretic response, maximum displacement and velocity demands, dissipated energy of the isolation system, and interstory drift for all levels of the superstructure. Experimental outcomes are compared to the related quantities returned by the same MDOF model [eq. (1)], by implementing the isolation force response according to the following hysteretic rule:

$$
F_{i s}^{n u m}=W_{i s}\left(\frac{u_{0}}{R_{e q}}+\mu\left(\frac{\dot{u}_{0}}{T S}\right) \cdot \tan h\left(\frac{\dot{u}_{0}}{v_{s}}\right)\right)
$$

Where:

$u_{0}$ is the translational degree of freedom located at the isolation level;

$W_{i s}$ is the vertical load applied to the device $(850 \mathrm{kN})$; 
$\nu_{s}$ is a hysteretic parameter which rules the slope of the friction coefficient trend at the transition at zero sliding velocity (the smaller the value, the sharper the transition); and

$R_{e q}$ and $\mu\left(\frac{\dot{u}_{0}}{T S}\right)$ are the equivalent radius of curvature and the coefficient of fiction, respectively.

According to the characterization curve (Figure 4), the friction coefficient has been assumed as a function of the actual velocity of the simulation, that is, the numerical velocity divided by the considered time scale $T S$, aiming at comparing results related to the same frictional response.

In addition, performances of the isolation system have been compared also to the dynamic behavior of a non-linear SDOF oscillator, according to the following system:

$$
M_{T O T} \cdot \ddot{u}_{0}+F_{i s}^{n u m} \cdot n_{i s}=-M_{T O T} \cdot \ddot{x}_{g}
$$

where $M_{T O T}$ represents the total mass of the isolated system; the same modeling strategy of the isolation force of the MDOF system has been assumed.

\section{Isolation Hysteretic Response}

In Figures 7, 8, hysteretic responses of the isolation system are shown, for time scales 32 and 8 , respectively. Results are compared between hybrid simulations and numerical MDOF dynamic system integration.

All inclined horizontal lines are related to a given friction coefficient value, and the inclination is associated to the equivalent radius of curvature of the device $(3.08 \mathrm{~m})$ : such a graphical representation of results allows analysis of the variation of the coefficient of friction during motion. It is possible to notice the velocity effect, that is, the common dependency of the friction coefficient on the sliding velocity of the simulation, which is represented by the real velocity value, divided by the time scale factor. Consequently, peak values of velocity are much lower than the real ones, and the transition phase between the slow and the fast friction coefficient parameters of the characterization curve can be better appreciated. In most cases, hysteretic loops of the purely numerical simulations fairly capture the hybrid experimental behavior of the isolation system, for both the considered time scale factors; the highest discrepancies can be detected for events \#6 and \#7, where the maximum displacement demand of the isolation level is overestimated by the numerical simulation. Thus, the adopted modeling strategy of the hysteretic response of the isolation system seems to lead to higher peak displacement values (maximum variation: approximately $+30 \%$ for time scale 32 ), even though in most cases hysteretic loops are almost overlapped with an accurate prediction of the real experimental force response of the physical device.

\section{Isolation System Peak Response Parameters}

In Figures 9-11, the peak response of the isolation system has been analyzed, in terms of maximum values of displacement, velocity, and force, respectively. Results are provided by comparing the considered response parameters returned by the SDOF and MDOF numerical oscillators and the hybrid simulations for all events; in addition, mean values among the selected earthquakes have been highlighted, in order to assess the accuracy of the response predictions computed by the equivalent linear elastic analysis.

Concerning the displacement response, for time scales 32 and 8 , the SDOF oscillator returns lower values, in comparison to both the MDOF model and the hybrid simulations, which look very similar, even though the MDOF model generally leads to higher displacement demands, as already noticed by analyzing hysteretic responses. On the other hand, if the mean value among the applied events is considered, both the numerical oscillators lead to significantly good results, especially for time scale 8. In addition, in all cases (SDOF, MDOF, and hybrid simulation), the mean displacement is lower than the reference value computed through the equivalent linear elastic analysis, according to the target spectrum provided by the standard code (approximately $150 \mathrm{~mm}$ ): thanks to the lower bound of the damping reduction factor of both acceleration and displacement spectra, higher displacement values can be achieved, and a safe definition of the displacement capacity of isolation devices can be made.

The peak velocity response of the isolation system is fairly captured by the MDOF numerical oscillator, in comparison to the hybrid simulation, and in some cases, also the SDOF model leads to reasonably good results. In the hybrid simulation, the real peak velocity of the physical device is bounded between 10 and $24 \mathrm{~mm} / \mathrm{s}$ for time scale 32 and between 45 and $96 \mathrm{~mm} / \mathrm{s}$ for time scale 8: the assumed friction coefficients for the equivalent linear elastic analyses exactly correspond to average values of velocity in the aforementioned ranges. This results into a fairly good estimation of the mean peak sliding velocity among the selected events for all the simulations (numerical SDOF and MDOF and experimental hybrid simulations), in comparison the value returned by the equivalent linear elastic analysis for both the adopted time scale factors.

Finally, concerning the peak force responses, variability among the considered simulations, both numerical and experimental, is significantly lower, and approximately the same results can be found for the single-event and mean results. Even though the physical device provides a highly non-linear force response, such a behavior can be accurately described and modeled through the adopted numerical hysteretic constitutive law.

\section{SUPERSTRUCTURE INTERSTORY DRIFT RESPONSE}

In Figure 12, the peak interstory drift profile is shown for the single-event and mean responses of both numerical MDOF model and the experimental hybrid simulations, by considering time scales 32 and 8 .

Numerical non-linear time history analyses of the MDOF model return approximately the same profiles of the hybrid simulation for both time scale factors. Mean profiles are represented by values approximately equal to $0.5 \%$, which can be considered as an upper bound to ensure a linear elastic 

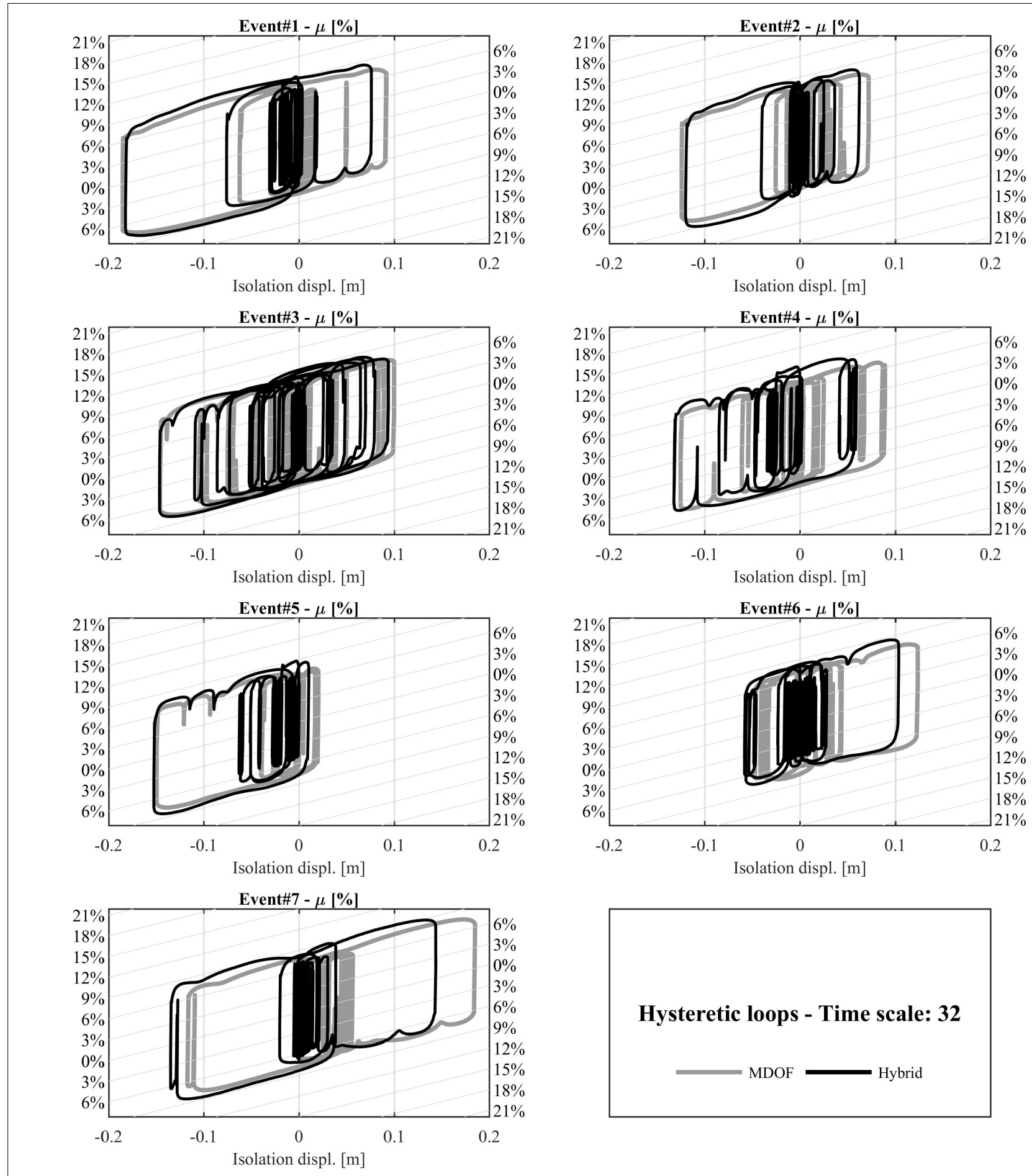

FIGURE 7 | Hysteretic response comparison-time scale 32.

behavior of the superstructure. Thus, also the overall behavior of the building in experimental hybrid simulations can be fairly approximated by assuming a proper non-linear constitutive law for the isolation level. Nonetheless, at some level of the superstructure, small variations can be detected.

\section{CONCLUDING REMARKS}

In this work, the experimental outcomes of hybrid earthquake simulations on a base-isolated building have been compared to results of purely numerical SDOF and MDOF oscillators, 

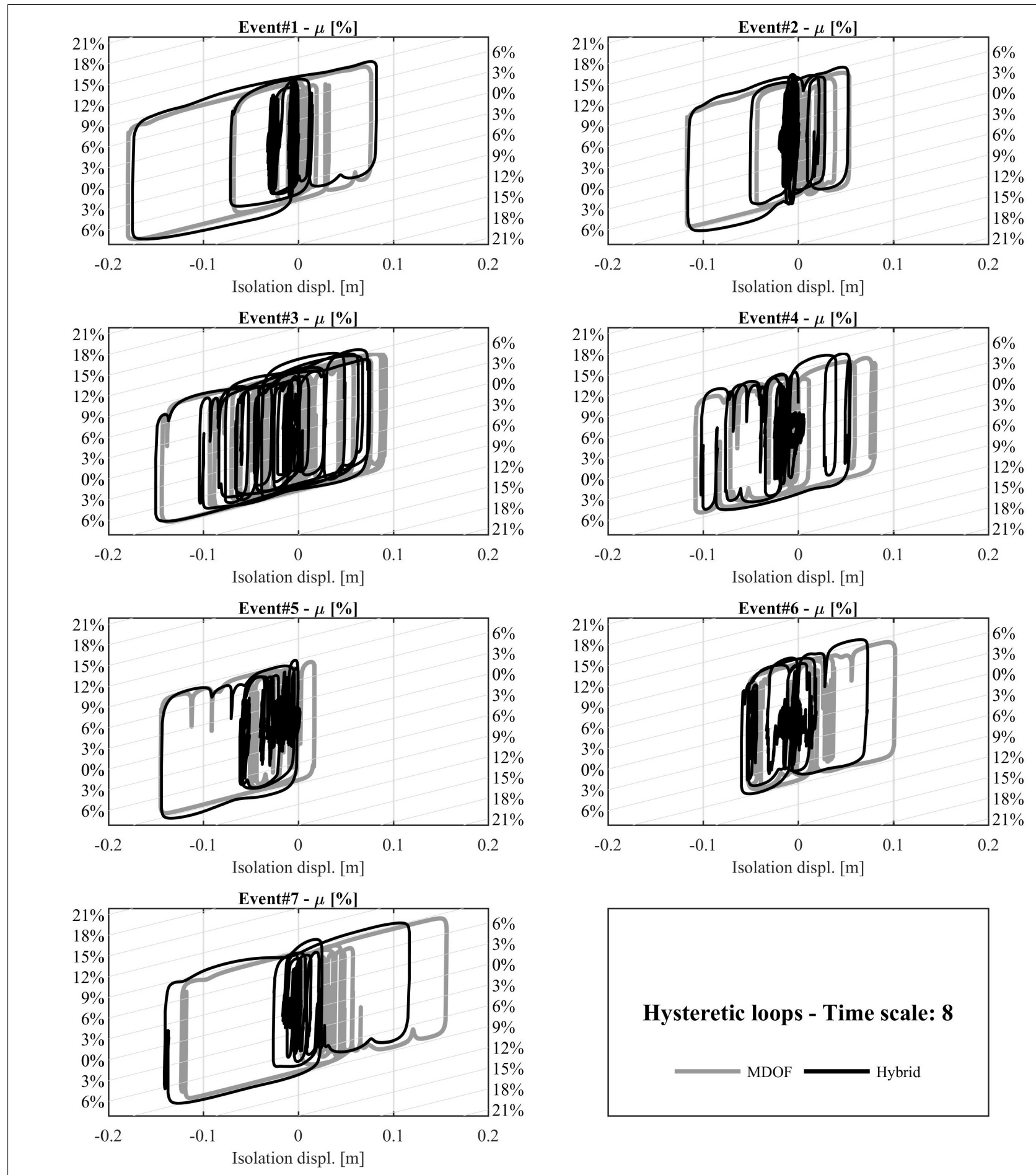

FIGURE 8 | Hysteretic response comparison-time scale 8.

which account for a proper non-linear constitutive law for the isolation system. Precisely, DCSS devices have been implemented for numerical simulations, whereas a physical device has been tested in the Bearing Tester System of the Laboratory of EUCENTRE Foundation in Pavia (Italy).
Tests have been carried out with a selection of seven natural seismic events, and spectrum compatibility has been ensured, by reducing the single-event discrepancy, with respect to the target spectrum provided by the standard code. A number of improvement of hybrid testing strategies 

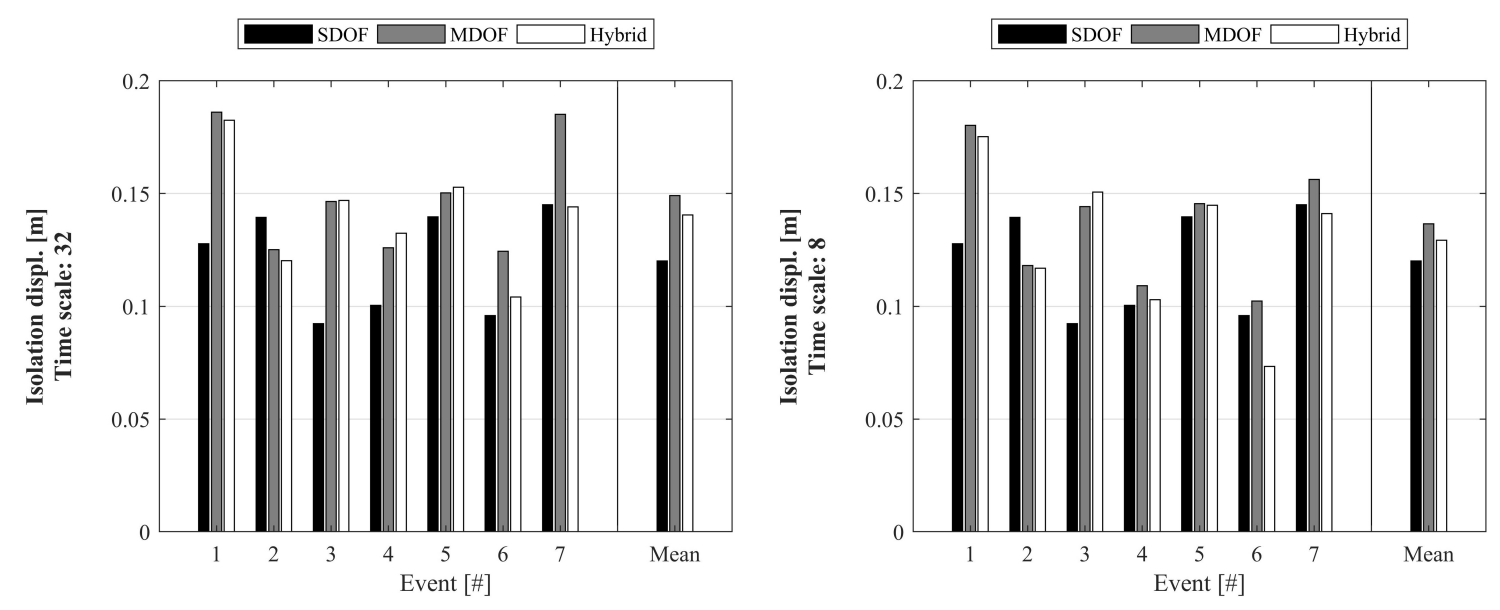

FIGURE 9 | Isolation displacement response-time scales 32 and 8.
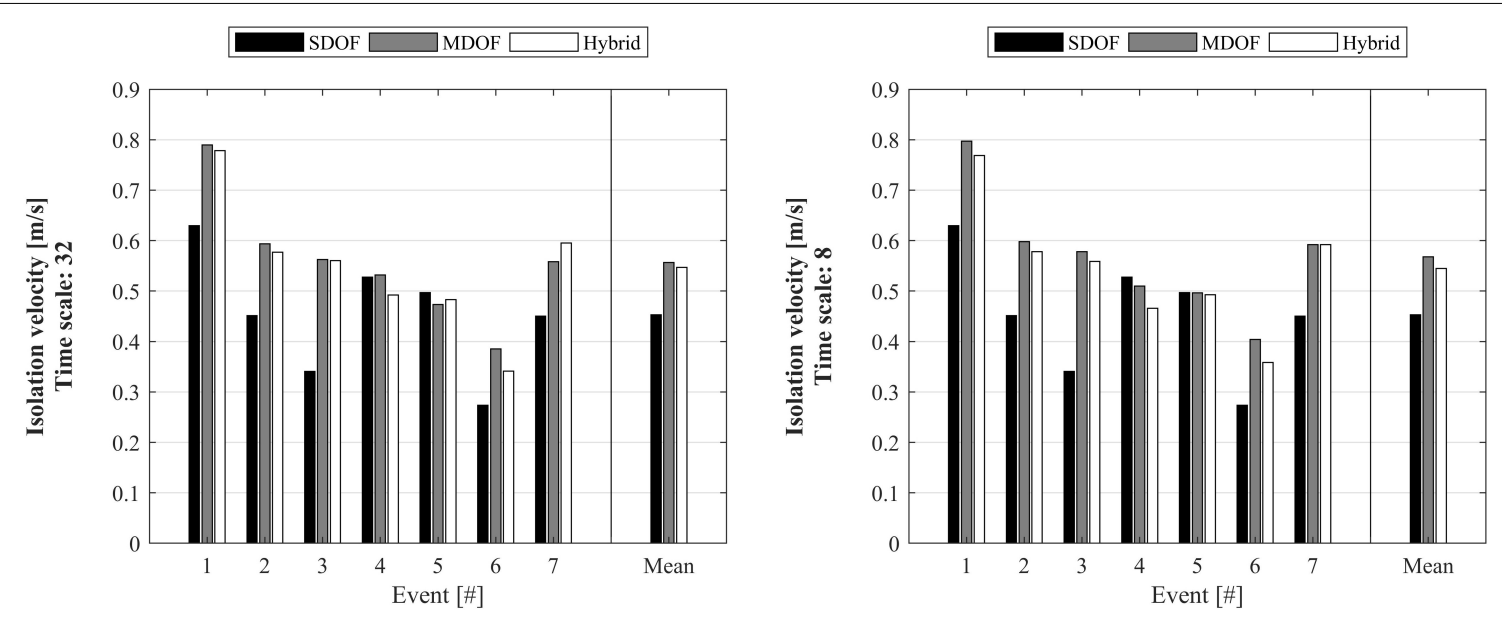

FIGURE 10 | Isolation velocity response-time scales 32 and 8.

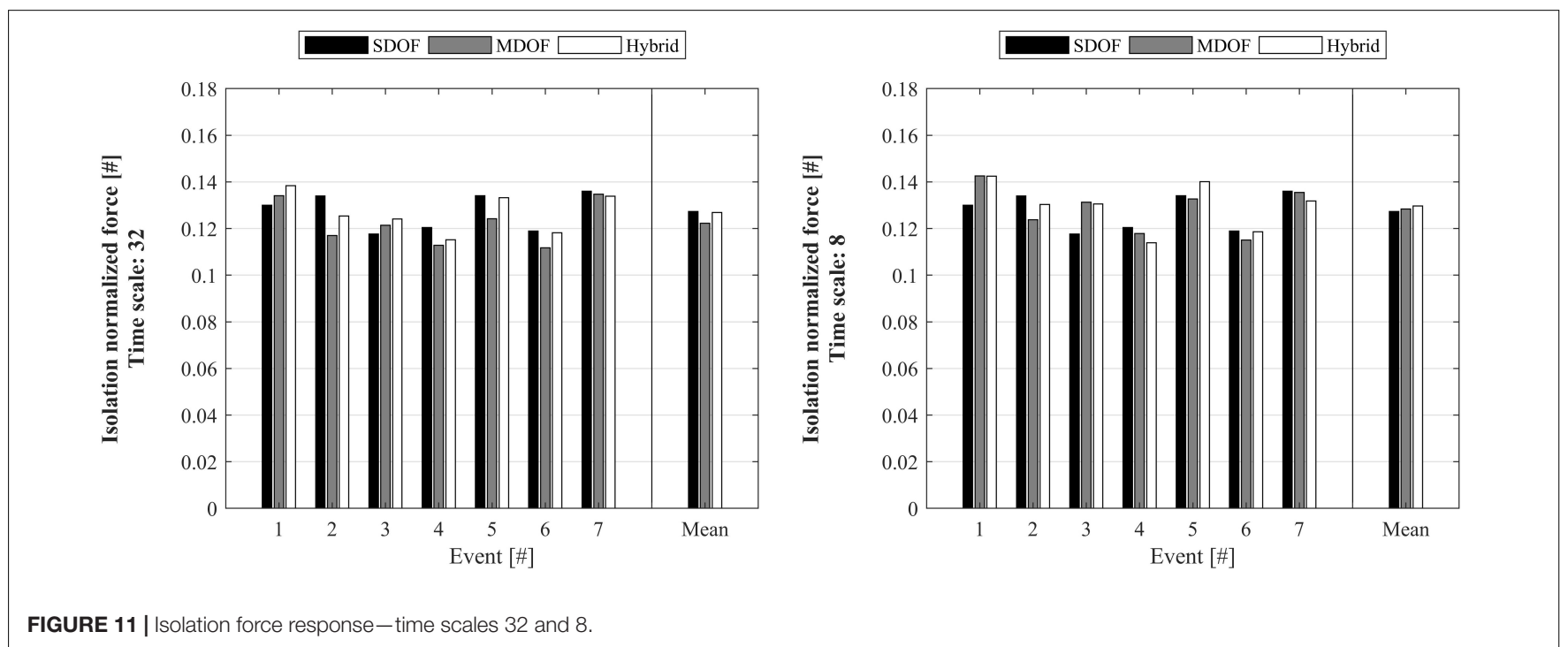



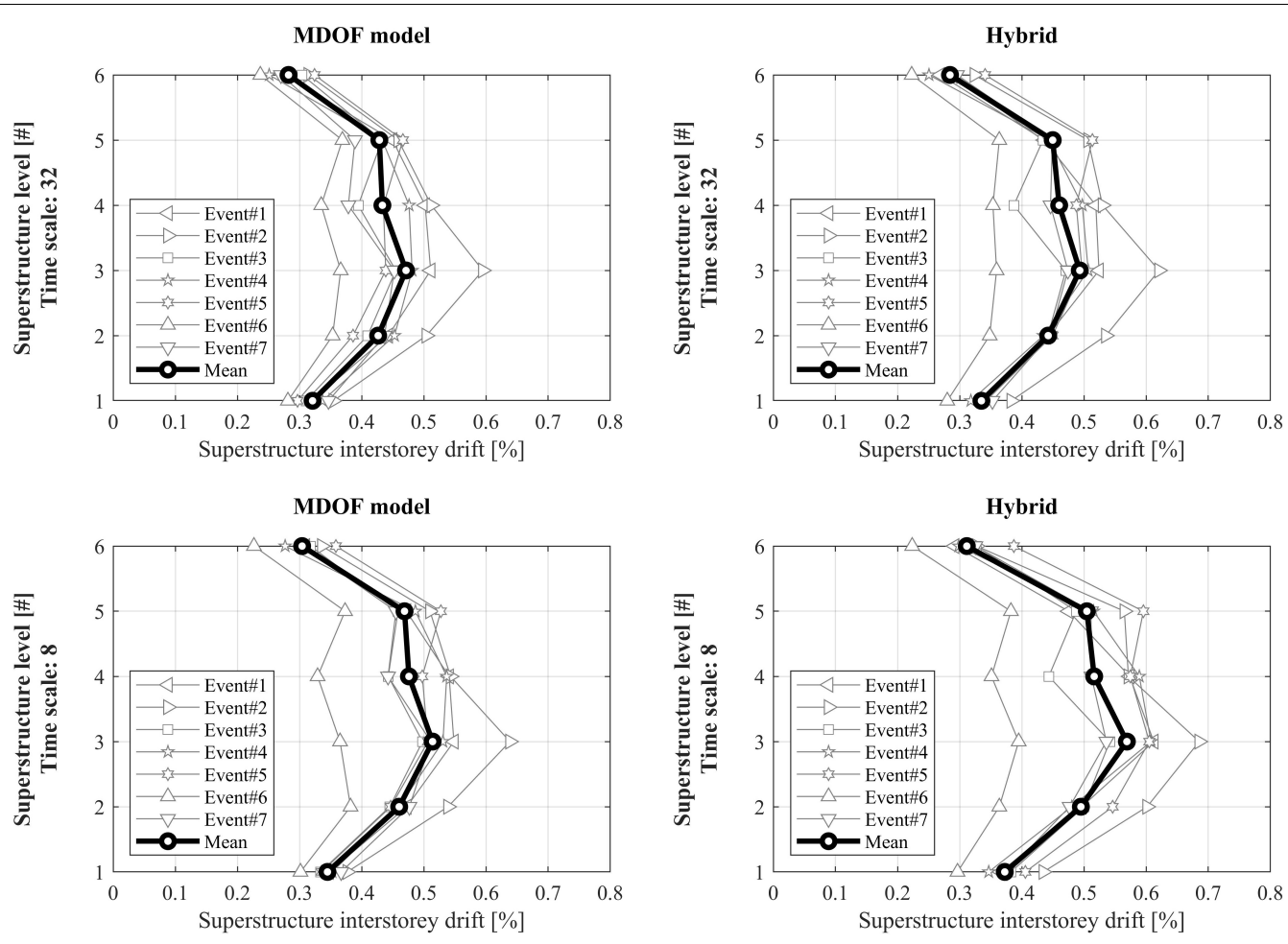

FIGURE 12 | Interstory drift response-time scales 32 and 8.

have been achieved, for the experimental assessment of base-isolated building:

- The numerical substructuring of hybrid simulations has been implemented as a statically condensed MDOF oscillator, which has the same dynamic properties as the full 3-D FEM of the case study structure, modeled by means of linear elastic structural elements;

- Hybrid simulations have been carried out by considering two individual time scale factors, namely 32 and 8 , in order to investigate different regions of the characterization curve, which correspond to different frictional properties, aiming at considering configurations closer to the realistic real-time simulation.

The outcomes of hybrid tests for all the selected events have been compared to numerical results of both simplified procedures and non-linear time history analyses. Precisely, we have the following:

- A preliminary estimation of the performance of the isolation system has been computed, through an equivalent linear elastic analysis, which accounts for the effective friction coefficient, according to a characterization curve obtained from dynamic tests, which have highlighted the common dependency of frictional properties of PTFEbased sliding materials on the velocity. Results have shown that the mean response of hybrid simulations can be fairly captured by the equivalent linear elastic analysis, which consequently represents a very useful tool for design of isolation systems, even though highly non-linear behaviors are considered.

- In addition, also non-linear time history analyses have been computed, by accounting for both SDOF and MDOF numerical oscillators. Both oscillators lead to comparable peak responses, even though the SDOF system returns lower displacements values. On the other hand, the MDOF system seems to provide overestimations of the more realistic displacement demands of the hybrid simulations. Similar conclusions can be drawn for sliding velocity response, and much lower discrepancies can be detected in the peak isolation force analysis. Concerning the isolation hysteretic response, for all events, the overall force of the device is properly captured by the numerical nonlinear constitutive law. Finally, the superstructure peak interstory drift profiles returned by the numerical MDOF model provide a good approximation of the more realistic maximum deformation related to hybrid tests.

The present testing campaign provides evidences that proper non-linear constitutive laws for isolation devices can effectively reproduce a realistic response of a baseisolated building, at least if the superstructure is linearly modeled; in addition, equivalent linear elastic analysis can be adopted for design purposes, in order to obtain safe estimations of the maximum displacement allowance of the implemented devices. More hybrid simulations 
could be carried out, by accounting for distributed (or lumped) plasticity for all structural elements of the superstructure, with time scale factors closer to real time, aiming at comparing simpler linear elastic responses to the outcomes of one of the most realistic testing techniques for base-isolated systems.

\section{DATA AVAILABILITY STATEMENT}

All datasets generated for this study are included in the article/supplementary material.

\section{AUTHOR CONTRIBUTIONS}

MF has defined and carried out all the numerical analyses and the post-processing of experimental data, by defining the

\section{REFERENCES}

Bonelli, A., Bursi, O. S., He, L., Magonette, G., and Pegon, P. (2008). Convergence analysis of a parallel inter eld method for heterogeneous simulations with dynamic substructuring. Int. J. Num. Methods Eng. 75, 800-825. doi: 10.1002/ nme. 2285

Bursi, O. S., Abbiati, G., Cazzador, E., Pegon, P., and Molina, F. J. (2017). Nonlinear heterogeneous dynamic substructuring and partitioned FETI time integration for the development of low-discrepancy simulation models. Int. J. Num. Methods Eng. 112, 1253-1291. doi: 10.1002/nme. 5556

Bursi, O. S., He, L., Bonelli, A., and Pegon, P. (2010). Novel generalized- methods for interfield parallel integration of heterogeneous structural dynamic systems. J. Comput. Appl. Math. 234, 2250-2258.

Calabrese, A., Strano, S., and Terzo, M. (2015). Real-time hybrid simulations vs shaking table tests: case study of a fibre-reinforced bearings isolated building under seismic loading. Struct. Contr. Health Monit. 22, 535-556. doi: 10.1002/ stc. 1687

Cardone, D., Conte, N., Dall'Asta, A., Di Cesare, A., Flora, A., Leccese, G., et al. (2017). "RINTC project: nonlinear analyses of Italian code-conforming base-isolated buildings for risk of collapse assessment," in COMPDYN 2017 Proceedings of the 6th International Conference on Computational Methods in Structural Dynamics and Earthquake Engineering, Rhodes Island

CEN (2018). Comité Européen de Normalisation TC 340, European Code UNI EN 15129:2018 Anti-Seismic Devices. Brussels: European Committee for Standardization.

Chopra, A. K. (1995). Dynamics of Structures Theory and Applications to Earthquake Engineering. Upper Saddle River, NJ: Prentice Hall.

Constantinou, M., Mokha, A., and Reinhorn, A. (1990). Teflon bearings in base isolation II: modeling. J. Struct. Eng. 116, 455-474. doi: 10.1061/(asce)07339445(1990)116:2(455)

D.M. 17/01/2018 (2018). Norme Tecniche per le Costruzioni, D.M. 17/01/2018. Italia: Gazzetta Ufficiale.

De Domenico, D., Ricciardi, G., and Benzoni, G. (2018). Analytical and finite element investigation on the thermo-mechanical coupled response of friction isolators under bidirectional excitation. Soil Dyn. Earthq. Eng. 106, 131-147. doi: 10.1016/j.soildyn.2017.12.019

Dolce, M., Cardone, D., and Croatto, F. (2005). Frictional behavior of steel-PTFE interfaces for seismic isolation. Bull. Earthq. Eng. 3, 75-99. doi: 10.1007/ s10518-005-0187-9

Fenz, D., and Constantinou, M. C. (2006). Behaviour of the double concave friction pendulum bearing. Earthq. Eng. Struct. Dyn. 35, 1403-1424. doi: 10.1002/eqe. 589

Furinghetti, M., and Pavese, A. (2017). Equivalent uniaxial accelerogram for CSSbased isolation systems assessment under two-components seismic events. research assumptions of hybrid simulations. IL has developed the algorithm for hybrid simulations and has managed the experimental part of the work. AP has managed the definition of the overall research, from both the experimental and the numerical points of view.

\section{FUNDING}

Part of the current work has been carried out under the financial support of the Italian Civil Protection, within the frameworks of the Executive Project 2017-2019 (Project 3Assessment of the seismic isolation of building structures through hybrid tests with numerical substructuring) and the national Research Project DPC-ReLUIS (National Network of Laboratories of Seismic Engineering) 2014-2018, Line 6Isolation and Dissipation.

Mechan. Based Des. Struct. Mach. 45, 282-295. doi: 10.1080/15397734.2017. 1281145

Furinghetti, M., Pavese, A., Quaglini, V., and Dubini, P. (2019a). Experimental investigation of the cyclic response of double curved surface sliders subjected to radial and bidirectional sliding motions. Soil Dyn. Earthq. Eng. 117, 190-202. doi: 10.1016/j.soildyn.2018.11.020

Furinghetti, M., Pavese, A., and Rizzo Parisi, E. (2019b). "Static condensation procedure of finite element models for fast non-linear time history analyses of base-isolated structures," in COMPDYN 2019 Proceedings of the 7th ECCOMAS Thematic Conference on Computational Methods in Structural Dynamics and Earthquake Engineering, Crete.

Gravouil, A., and Combescure, A. (2001). Multi-time-step explicit - implicit method for non-linear structural dynamics. Int. J. Num. Method Eng. 50, 199-225. doi: 10.1002/1097-0207(20010110)50:1<199::aid-nme132> 3.0.co; 2 -a

Hakuno, M., Shidawara, M., and Hara, T. (1969). Dynamic destructive test of a cantilever beam controlled by an analog computer. Trans. Jpn. Soc. Civil Eng. 171, 1-9. doi: 10.2208/jscej1969.1969.171_1

Iervolino, I., Galasso, C., and Cosenza, E. (2009). REXEL: computer aided record selection for code-based seismic structural analysis. Bull. Earthq. Eng. 8, 339362. doi: 10.1007/s10518-009-9146-9141

Kumar, M., Whittaker, A. S., and Constantinou, M. C. (2015). Characterizing friction in sliding isolation bearings. Earthq. Eng. Struct. Dyn. 44, 1409-1425. doi: 10.1002 /eqe. 2524

Lanese, I. (2012). Development and Implementation of an Integrated Architecture for Real-Time Dynamic Hybrid Testing in the Simulation of Seismic Isolated Structures. Ph.D. Dissertation, Rose School, Pavia.

Lanese, I., Pavese, A., and Furinghetti, M. (2018). "Hybrid testing of seismic isolated structures: facing time and geometry scaling issues," in Proceedings of 16th European Conference on Earthquake Engineering, Thessaloniki.

Lomiento, G., Bonessio, N., and Benzoni, G. (2013). Concave sliding isolator's performance under multi-directional excitation. Ingegneria Sismica 30, 17-32.

Mosqueda, G., Whittaker, A. S., and Fenves, G. L. (2004). Characterization and modeling of friction pendulum bearings subjected to multiple components of excitation. J. Struct. Eng. 130, 433-442. doi: 10.1061/(asce)0733-9445(2004) 130:3(433)

Pavese, A., Furinghetti, M., and Casarotti, C. (2019). Investigation of the consequences of mounting laying defects for curved surface slider devices under general seismic input. J. Earthq. Eng. 23, 377-403. doi: 10.1080/13632469.2017. 1323046

Pegon, P., and Magonette, G. (2002). Continuous PSD Testing with Nonlinear Substructuring: Presentation of a Stable Parallel Inter-Field Procedure. Technical Report 1.02.167. Ispra: European Commission. 
Pegon, P., and Pinto, A. (2000). Pseudo-dynamic testing with substructuring at the ELSA Laboratory. Earthquake Eng. Struct. Dynam. 29, 905-925. doi: 10.1002/ 1096-9845(200007)29:7<905::aid-eqe941>3.0.co;2-p

Peloso, S., Pavese, A., and Casarotti, C. (2012). Eucentre trees lab: laboratory for training and research in earthquake engineering and seismology. Geotech. Geol. Earthquake Eng. 20, 65-81. doi: 10.1007/978-94-007-1977-4_4

Quaglini, V., Bocciarelli, M., Gandelli, E., and Dubini, P. (2014). Numerical assessment of frictional heating in sliding bearings for seismic isolation. J. Earthquake Eng. 18, 1198-1216. doi: 10.1080/13632469.2014. 924890
Conflict of Interest: The authors declare that the research was conducted in the absence of any commercial or financial relationships that could be construed as a potential conflict of interest.

Copyright (C) 2020 Furinghetti, Lanese and Pavese. This is an open-access article distributed under the terms of the Creative Commons Attribution License (CC BY). The use, distribution or reproduction in other forums is permitted, provided the original author(s) and the copyright owner(s) are credited and that the original publication in this journal is cited, in accordance with accepted academic practice. No use, distribution or reproduction is permitted which does not comply with these terms. 